\title{
Molecular mechanisms of inhibition of photocarcinogenesis by silymarin, a phytochemical from milk thistle (Silybum marianum L. Gaertn.) (Review)
}

\author{
MUDIT VAID $^{1}$ and SANTOSH K. KATIYAR ${ }^{1-3}$ \\ ${ }^{1}$ Department of Dermatology and ${ }^{2}$ Comprehensive Cancer Center, University of Alabama at \\ Birmingham; ${ }^{3}$ Birmingham Veterans Affairs Medical Center, Birmingham, AL 35294, USA
}

Received January 4, 2010; Accepted February 25, 2010

DOI: 10.3892/ijo_00000586

\begin{abstract}
Changes in life style over the past several decades including much of the time spent outdoors and the use of tanning devices for cosmetic purposes by individuals have led to an increase in the incidence of solar ultraviolet (UV) radiation-induced skin diseases including the risk of skin cancers. Solar UV radiations are considered as the most prevalent environmental carcinogens, and chronic exposure of the skin to UV leads to squamous and basal cell carcinoma and melanoma in human population. A wide variety of phytochemicals have been reported to have substantial anticarcinogenic activity because of their antioxidant and antiinflammatory properties. Silymarin is one of them and extensively studied for its skin photoprotective capabilities. Silymarin, a flavanolignan, is extracted from the fruits and seeds of milk thistle (Silybum marianum L. Gaertn.), and has been shown to have chemopreventive effects against photocarcinogenesis in mouse tumor models. Topical treatment of silymarin inhibited photocarcinogenesis in mice in terms of tumor incidence, tumor multiplicity and growth of the tumors. Wide range of in vivo mechanistic studies conducted in a variety of mouse models indicated that silymarin has anti-oxidant, anti-inflammatory and immunomodulatory properties which led to the prevention of photocarcinogenesis in mice. This review summarizes and updates the photoprotective potential of silymarin with the particular emphasis on its in vivo mechanism of actions. It is suggested that silymarin may favorably supplement sunscreen protection,
\end{abstract}

Correspondence to: Dr Santosh K. Katiyar, Department of Dermatology, University of Alabama at Birmingham, 1670 University Boulevard, Volker Hall 557, P.O. Box 202, Birmingham, AL 35294, USA

E-mail: skatiyar@uab.edu

Key words: antioxidant, oxidative stress, inflammation, silymarin, photocarcinogenesis, interleukins, ultraviolet radiation, contact hypersensitivity and may be useful for skin diseases associated with solar UV radiation-induced inflammation, oxidative stress and immunomodulatory effects.

\section{Contents}

1. Introduction

2. Multistage model of UV radiation-induced skin cancer

3. Sunscreen and UV-carcinogenesis

4. Skin photoprotection by phytochemicals

5. Silymarin: source and composition

6. Silymarin inhibits UV radiation-induced skin carcinogenesis

7. Mechanisms of chemoprevention of photocarcinogenesis by silymarin

8. Future prospects of silymarin in skin photoprotection

\section{Introduction}

Changes in life style over the past several decades have provided individuals with greater amounts of time for recreational activities and much of this time has been spent outdoors. As a result there has been alarming increase in the incidence of solar ultraviolet (UV) radiation-induced skin disorders including skin cancers. Overexposure of the skin to UV radiation causes sunburn, inflammation, oxidative stress, DNA damage and suppression of immune system which leads to premature aging of the skin, basal cell and squamous cell carcinoma, and melanoma (1-4).

Epidemiological and statistical analyses reveal that more than 1.3 million new cases of non-melanoma skin cancers are diagnosed every year in the United States alone. However, these numbers are probably underestimates as many cases of skin cancers are treated or removed in clinics without being reported to cancer registries. Thus, solar UV radiation is considered as the most prevalent environmental carcinogen $(5,6)$. The incidence of skin cancer has been increasing dramatically, and this increase is expected to continue as the population ages and larger amounts of UV radiation reach the surface of the Earth because of depletion of the ozone layer (1-3). UVB (290-320 nm) component of the solar UV 


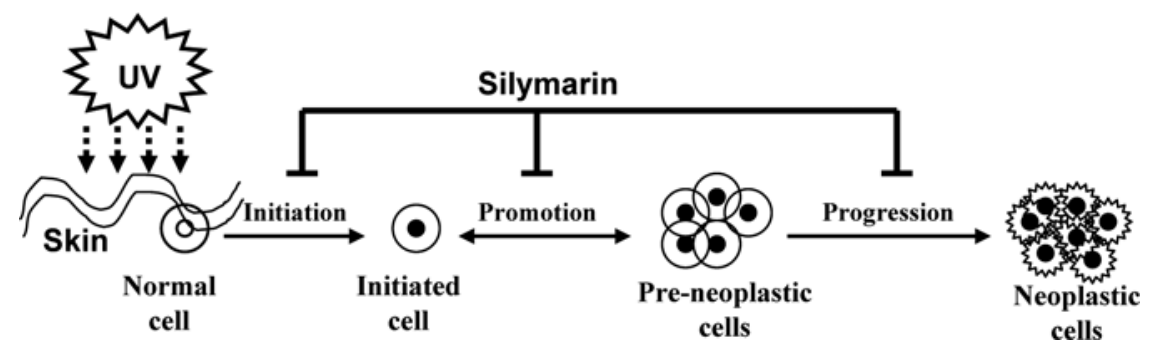

Figure 1. Schematic representation of UV radiation-induced multi-stage skin carcinogenesis, showing initiation, promotion and progression stages. In the initiation stage genetic changes occurs in the cells. At the promotion stage, additional UV irradiation leads to the clonal expansion of initiated cells. Tumor progression stage involves the transformation of the benign tumor into an invasive and potentially metastatic malignant tumor. Silymarin can reverse, inhibit or retard the process of skin carcinogenesis at one or at all the stages of carcinogenesis.

spectrum can act as tumor initiator, tumor promoter and as a complete carcinogen by damaging cellular macromolecules such as DNA, proteins and lipids $(1,4,7)$.

It is well documented that UV irradiation to skin induced the generation of reactive oxygen species (ROS), such as singlet oxygen, peroxy radicals, superoxide anion and hydroxyl radicals which creates a state of oxidative stress in the target cells. Oxidative stress results when the formation of ROS exceeds the antioxidant defense ability of the target cells. ROS can also act as tumor initiator and tumor promoter by damaging cellular macromolecules, and by activating cellsignaling molecules (8-11). Thus, oxidative stress or ROS has been implicated in many disease processes including skin cancer (4).

It has been recognized that UV-induced DNA damage, predominantly in the form of cyclobutane pyrimidine dimers (CPD), plays an important role in immune suppression and skin cancer initiation. Accumulation of damaged DNA lesions results in mutations in critical genes and contributes to the development of nonmelanoma skin cancers. Mammalian cells possess efficient mechanisms to preserve genomic stability, however, if the damage is too severe after a higher dose of UV irradiation, cells have a mechanism to trigger apoptosis or programmed cell death to prevent the propagation of the damaged DNA $(12,13)$. Thus, CPD are implicated in UV-induced suppression of immune system $(14,15)$ and carcinogenesis $(15,16)$, and there is a close association between UV-induced suppression of immune system and carcinogenesis $(17,18)$. The DNA strand break, thymine glycols and 8-hydroxyguanine are the forms of oxidative DNA damage. Strand breakage of DNA is induced by both UVB (290-320 nm) and UVA (320-400 nm) but to a greater extent by UVA exposure (19).

Statistical analysis reveals that the average annual UV dose that an American normally receive in a year is $~ 2,500$ $3,300 \mathrm{~mJ} / \mathrm{cm}^{2}$. An average female is exposed to 2,200 $\mathrm{mJ} / \mathrm{cm}^{2}$ and a male $2,800 \mathrm{~mJ} / \mathrm{cm}^{2}$ each year with an additional exposure of $\sim 800 \mathrm{~mJ} / \mathrm{cm}^{2}$ of solar UVB radiation during a conservative vacation $(20,21)$. According to the current projections, one in five Americans will develop at least one non-melanoma skin cancer during their lifetime. The cost of treating non-melanoma skin cancer is estimated to be in excess of US\$650 million/year (www.cancer.org/statistics), therefore prevention of skin cancer caused by chronic UV exposure is a plausible option.
A number of methods which include, a) avoiding direct exposure to the sun light between 10:00 a.m. to 4:00 p.m., or b) protecting exposed areas of skin by wearing hats/clothing to adequately cover the body from direct sunlight or using sun screen lotions with sun protection factor $>16$, have been suggested to prevent direct exposure from the sun and thus to minimize the occurrence and risk of skin cancer. While not to deny the importance of these preventive measures, their practical compliance is an issue. Especially with studies on modest efficacy of sunscreens (22), particularly on skin that has been previously damaged by UV exposure and the fact that very large amounts of sunscreen lotions are needed by photosensitive patients to achieve full SPF value present on label of sunscreen lotions (23). Therefore, there is an urgent need to look for alternative preventive approaches.

\section{Multistage model of UV radiation-induced skin cancer}

The main etiological factor for the non-melanoma skin cancer is the chronic exposure of the skin to solar UV radiation. The UV spectrum of sun light is subdivided into three parts based on their wavelengths; UVA (320-400 nm), UVB (280-320 nm) and UVC (200-280 nm). The UVC part of sunlight is filtered out by stratospheric ozone layer, the only wavelengths that reach the surface of the earth are UVA (90-99\%) and UVB (1-10\%) based on the geographic conditions. The process of skin carcinogenesis is divided into three distinct stages: initiation, promotion, and progression (Fig. 1).

The tumor initiation stage is an irreversible process associated with the genotoxic damage of cellular DNA upon exposure to endogenous or exogenous carcinogens, such as UV radiation. In contrast, tumor promotion is a long-lasting reversible process characterized by clonal expansion of initiated cells to form a benign tumor with rapidly proliferating potential. This stage requires repeated influence of the promoting agent to give rise to a benign tumor. Tumor progression involves the transformation of the benign tumor into an invasive and potentially metastatic malignant tumor (Fig. 1). Both UVA and UVB irradiation can act in mouse skin model as a complete carcinogen. It means that these wavelengths of solar light can function as initiation, promotion and progression factors. However, UVA is a relatively weak complete carcinogen because of its weak activity as an initiating agent, but it is a relatively potent tumor-promoting agent (4). 


\section{Sunscreens and UV carcinogenesis}

The use of sunscreen is widely advocated as a means of reducing UV-induced skin cancer risk. This advice is largely based on extrapolation from animal studies, as it is difficult to evaluate long-term protection in humans. Limited studies indicate that sunscreens can inhibit actinic keratoses that are considered as precursors of squamous cell carcinoma $(24,25)$. A study has shown that daily use of a SPF 16 sunscreen, over a period of 4.5 years, reduced the total number of squamous cell carcinoma by $40 \%$ but not the number of people with the tumor. No protective effect was seen for basal cell carcinoma after the use of SPF 16 sunscreen (22).

Some studies show that the use of sunscreen is associated with an increased risk of melanoma (26). It also has been noticed that sunscreens inadequately protect against UVinduced free radicals in skin which are implicated in skin aging and the risk of melanoma (27). Moreover, it is not simple to find an effective sunscreen which can provide full protection against ultraviolet spectrum. Additionally, sunscreen ingredients may become free radicals themselves when activated by UV irradiation (28), and sunscreen chemicals may be absorbed into the skin (29) to potentially cause harm. Therefore, the use of phytochemicals which possess antioxidants, anti-inflammatory and/or immunomodulatory effects may prove highly beneficial for the reduction of solar UV radiation induced skin photodamage including the risk of melanoma and nonmelanoma skin cancers.

\section{Skin photoprotection by phytochemicals}

Since ancient times plant products have been used for enhancing skin health and its protection from environmental factors including sunlight. Naturally occurring phytochemicals constitute a wide variety of agents which are distributed in plants, including fruits, vegetables, seeds, nuts, flowers and bark. The use of phytochemicals is receiving constant interest to protect skin from the adverse biological effects of solar UV radiation. These phytochemicals hold promise to be used as a complementary and alternative medicine for various skin disorders. The use of phytochemicals in skin care products has received attention by researchers, industry, consumers and the news media particularly for the protection of human skin from the damaging effects of external environmental stimuli, including solar UV radiation. The phytochemicals which have anti-oxidant, anti-inflammatory and immunomodulatory effects are beneficial and important in terms of photoprotective effects to the skin.

Several studies have demonstrated the efficacy of naturally occurring phytochemicals in animal models related to protection against UV radiation-induced inflammation, oxidative stress, immunosuppression and skin cancer. These phytochemicals are vitamin E (30), green tea polyphenols $(31,32)$, garlic $(33)$, ginger $(34)$, silymarin $(7,35)$, vitamin C (30), all-trans retinoic acid (36), proanthocyanidins from grape seeds (37) and lutein (carotenoids) (38). Since oxidants play an important role in several skin disorders including the initiation and promotion stages of multistage skin carcinogenesis, the antioxidants can be targeted for intervention at the initiation, promotion or progression stages of

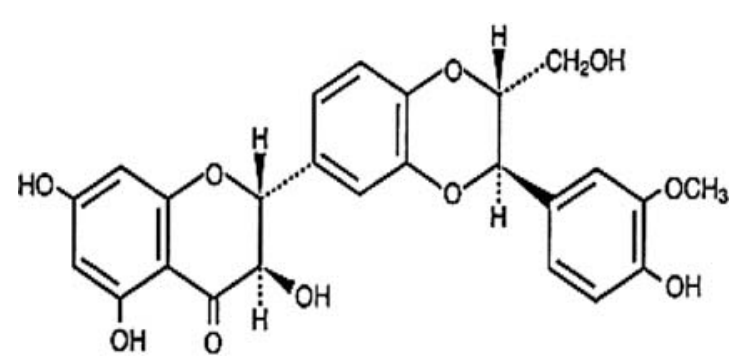

Figure 2. Chemical structure of silibinin, the major and most biologically active component of silymarin.

multistage skin carcinogenesis or other age-related skin disorders like premature aging of the skin $(39,40,41)$. Therefore, regular intake of antioxidants has been suggested as an important preventive strategy against the toxic effects of mutagenic and carcinogenic agents (42-45). Several investigations have demonstrated the photoprotective effects of polyphenols from green tea $(31,32,40,44)$, silymarin from milk thistle $(7,35)$ and proanthocyanidins from grape seeds against UV radiation-induced skin carcinogenesis in animal models (37). The present review specifically highlights the mechanism of inhibition of UV radiation-induced skin cancer or photocarcinogenesis by silymarin.

\section{Silymarin: source and composition}

Silymarin, a flavanolignan, extracted from the fruits and seeds of the plant milk thistle (Silybum marianum L. Gaertn.) (46). Milk thistle belongs to the family of Asteraceae and primarily is an indigenous plant of Mediterranean region and southwest Europe. Silymarin is a mixture of mainly three flavonolignans, silybin (silibinin), silydianin, and silychristin $(47,48)$. Silibinin is the major $(70-80 \%)$ and most active biological component of silymarin (Fig. 2). The seeds of milk thistle have been used for the last 2,000 years for liver diseases.

Pharmacological studies revealed that silymarin is non-toxic even at higher physiological doses, which suggests its safer use for humans (49). Laboratory studies suggest that there is no significant difference between silymarin and silibinin in terms of chemopreventive or biological activities conducted in several in vitro and in vivo cancer models $(50,51)$. Silymarin has been primarily used in liver disorders including hepatitis, alcoholic liver diseases and cirrhosis $(49,52,53)$ and is also useful for toxin-induced liver toxicity, including poisoning from a fungus called death cap mushroom (Amanita phalloides) (54). Based on the anti-oxidant and anti-inflammatory activity of silymarin, the chemopreventive effect of silymarin has been tested and determined using animal models of chemical carcinogenesis and photocarcinogenesis $(7,55,56)$. Since then extensive mechanism-based chemopreventive studies have been performed in vitro in cell culture and in vivo animal models to assess the efficacy of silymarin. Further, as UVinduced inflammation, oxidative stress and immunosuppression are primarily implicated in UV radiation-induced skin carcinogenesis; we will discuss the effect of silymarin on these mechanistic pathways or targets. 


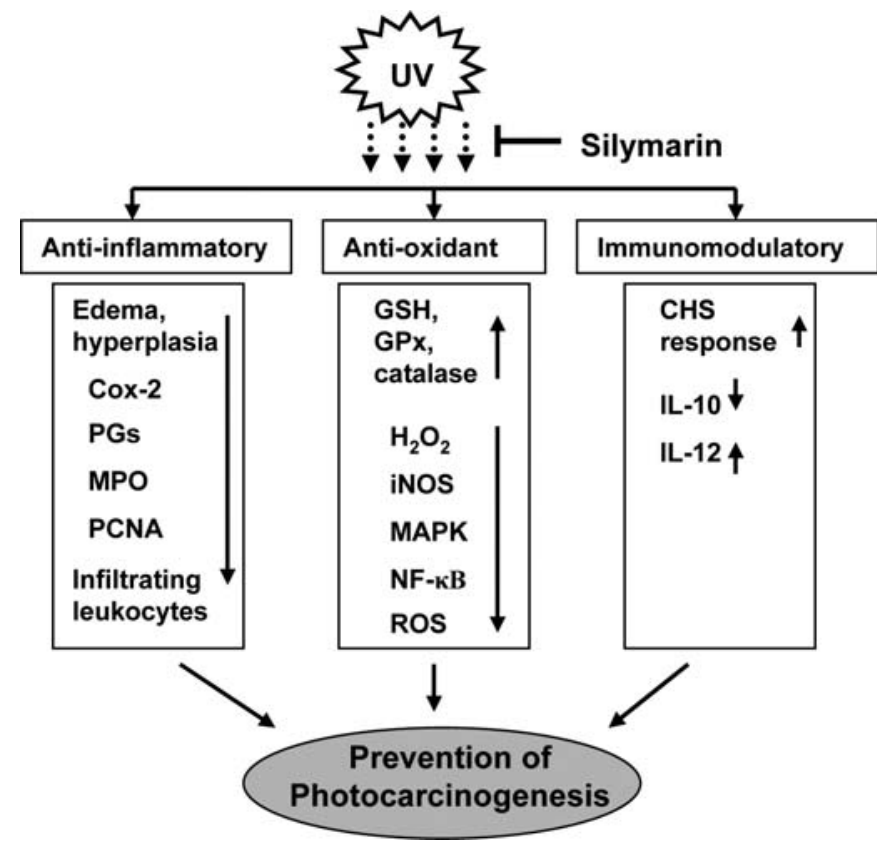

Figure 3. Molecular mechanisms and targets of silymarin in prevention of photocarcinogenesis. Upward arrows $(\uparrow)$ indicate the enhancement or stimulation and downward arrows $(\downarrow)$ indicates inhibition or downregulation of target molecules.

\section{Silymarin inhibits UV radiation-induced skin carcino- genesis}

It is well established that multiple environmental and genetic factors contribute to the development of skin cancers however; the most important is chronic exposure of the skin to solar UV radiation. Epidemiological and clinical studies have implicated solar UV radiation as the major etiological agent in the development of cutaneous malignancy $(1-3,57,58)$. Cancer chemoprevention strategies may have the ability to prevent or delay the occurrence of cancer in high-risk populations, such as those with pre-malignant lesions, previous resected cancers or exposure to high levels of environmental carcinogens. Nonmelanoma skin cancers, including basal and squamous cell carcinomas, represent the most common malignant neoplasms in humans $(1,2,57)$.

Using the SKH-1 hairless mouse model, we showed that topical application of silymarin to mouse skin prevented UVBinduced skin carcinogenesis in terms of tumor incidence (percent mice with tumors), tumor multiplicity and tumor size compared to non-silymarin treated mice (7). The anticarcinogenic effect of silymarin was pronounced against all the stages of photocarcinogenesis such as UV-induced tumor initiation, tumor promotion, and complete carcinogenesis protocols (including initiation and promotion) (7). Silibinin, which is a major component of silymarin, was also found to inhibit photocarcinogenesis in SKH-1 hairless mice when applied topically (59). Dietary effect of both silymarin and silibinin was also examined against UV radiation-induced skin tumor development in mice. It was found that dietary silibinin also prevented photocarcinogenesis in terms of tumor multiplicity, tumor volume/mouse throughout the experiment, however; only a moderate chemopreventive effect was observed on tumor incidence. Additionally, the treatment of silymarin or silibinin also increased latency period of tumor appearance specifically in complete UVB photocarcinogenesis protocol. The increment in latency period reveals the diseasefree survival period of the mice.

\section{Mechanisms of chemoprevention of photocarcinogenesis by silymarin}

The development of a new chemopreventive or chemotherapeutic agent requires understanding of the molecular mechanism or targets which are affected during the chemoprevention of cancer process. To determine the mechanisms of prevention of skin cancer by silymarin or silibinin, studies were performed to define anti-photocarcinogenic activity using in vitro and in vivo systems. These studies were focused on the effect of silymarin on UVB-induced inflammatory effect, oxidative stress, suppression of immune responses and DNA damaging effects in the skin because the alterations in these biochemical markers by UVB radiation results in several adverse biological effects which leads to photocarcinogenesis. Detailed studies are summarized below:

Anti-oxidant effect of silymarin. Skin is easily accessible and constantly exposed to free radical-generating agents such as solar UV radiation, ozone and other environmental pollutants (4). Studies have shown the involvement of oxidative stress in skin carcinogenesis (4), and also demonstrated that a sophisticated enzymatic and non-enzymatic antioxidant defense system including catalase, superoxide dismutase and glutathione peroxidase counteracts and regulates overall ROS levels to maintain physiological homeostasis. Elevated levels of ROS are detrimental to target cells.

It has been shown that UV irradiation of SKH-1 hairless mice results in a significant depletion of antioxidant defense enzymes in the skin (60). Topical treatment of silymarin to SKH-1 hairless mice resulted in inhibition of UVB-induced intracellular production of $\mathrm{H}_{2} \mathrm{O}_{2}$, a stable oxidant, in both epidermis and dermis compared with non-silymarin treated animals (35) (Fig. 3). It has been found that UVB-induced oxidative stress was significantly inhibited by silymarin through the inhibition of UV-induced infiltration of leukocytes. Skin exposure to UVB radiation also induced the expression of inducible nitric oxide synthase and resulted in increased amount of nitric oxide production compared to that of nonUVB irradiated mice. Topical treatment of silymarin affords significant protection against UVB-induced expression of inducible nitric oxide synthase and subsequently inhibition of nitric oxide production (35).

Katiyar et al found that CD11 $\mathrm{b}^{+}$infiltrating cells in UVBirradiated skin are the major source of oxidative stress (61). Silymarin inhibits UVB-induced oxidative stress through inhibition of infiltrating CD11 $\mathrm{b}^{+}$cell types. Similar effect was also found when UVB-exposed mice were intraperitoneally treated with antibodies specific to CD11b. Thus confirming that silymarin inhibits UVB-induced oxidative stress in mouse skin through targeting CD11 $\mathrm{b}^{+}$cell type (61). Sobodova et al have shown that pretreatment of HaCaT cells with silymarin or its derivatives suppresses UVB-induced photodamage including the reduction in the levels of glutathione, total ROS 
production and lipid peroxidation in HaCaT cells $(62,63)$. These results indicated the antioxidant nature of silymarin which have a role in photoprotection of oxidative stressassociated skin disorders including skin cancer. UV-induced oxidative stress mediates activation of mitogen-activated protein kinases (MAPK) and nuclear factor- $\kappa \mathrm{B}(\mathrm{NF}-\kappa \mathrm{B})$ signaling pathways, and these pathways have been implicated in tumor development, as summarized in Fig. 3 (64).

In vivo studies conducted in mouse models indicate that topical application of silibinin inhibits UVB-induced expression levels of ERK1/2 and p38 proteins of MAPK family, and subsequently inhibited the activation of NF- $\mathrm{NB} /$ p65 through inhibition of degradation of I $\mathrm{B} \alpha$ and activation of IKK $\alpha$. As NF-кB-targeted genes play critical roles in inflammation and cellular proliferation, the inhibitory effect of silibinin on NF-кB is significant (65). Together, these antioxidant activities demonstrate that silibinin or silymarin have the ability to protect the skin from the adverse biological effects of UVB radiation via modulation of the MAPK and $\mathrm{NF}-\kappa \mathrm{B}$ signaling pathways and provide a molecular basis for the anti-carcinogenic effect of silymarin/silibinin in an in vivo animal model.

Anti-inflammatory effects of silymarin. UV-induced inflammatory responses, which are characterized by increased blood flow and vascular permeability, result in the development of edema, erythema, hyperplastic responses, and increases in the levels of cyclooxygenase-2 (COX-2) and prostaglandin (PG) metabolites $(4,66-68)$. UV-induced inflammation is considered as an early and important event in tumor promotion or the growth of skin tumors. Chronic inflammation plays an important role in all three stages of tumor development, i.e., initiation, promotion and progression (4). Therefore, the control on UVB-induced inflammatory responses is considered as an important strategy to prevent skin cancer risk. Topical treatment of silymarin inhibits UVB-induced edema and hyperplastic response in SKH-1 hairless mouse skin when compared with that of non-silymarin treated but UVB exposed mouse (7). It was observed that exposure of both mouse and human skin with UVB induces infiltration of inflammatory leukocytes which are the major source of oxidative stress such as $\mathrm{H}_{2} \mathrm{O}_{2}$ and nitric oxide production $(32,69,70)$. UV induced infiltration begins significantly after $24 \mathrm{~h}$ of UV irradiation and persists up to $72 \mathrm{~h}$ compared with the non-UV irradiated control mice. Treatment of silymarin significantly inhibits UV-induced infiltration of leukocytes which further supports the anti-inflammatory effect of silymarin (Fig. 3).

Myeloperoxidase is commonly used as a marker of leukocyte infiltration. The increase in myeloperoxidase activity after UVB exposure indicates an influx of leukocytes to the inflamed skin. Topical treatment of silymarin resulted in significant reduction in myeloperoxidase activity both in epidermis and dermis of UV-exposed mouse skin (35). Reduction in myeloperoxidase activity by silymarin suggests the inhibition of UV-induced infiltration of inflammatory leukocytes, and thus anti-inflammatory effect of silymarin in this mouse model. Topical application of silymarin also inhibits UVB-induced expression of COX-2 and its prostaglandin metabolites in the skin (7). These studies suggest that anti-photocarcinogenic activity of silymarin is associated with the inhibition of UVB-induced inflammation and inflammatory mediators in the mouse skin. It is suggested that silymarin may prove to be a useful chemopreventive agent against UVB radiation induced inflammationassociated skin diseases including melanoma and nonmelanoma skin cancers in humans, and therefore, more detailed studies with particular emphasis on molecular mechanisms could lead to new strategies for the prevention of inflammation-associated skin diseases in humans.

Silymarin inhibits UVB-induced immune suppression. Skin exposure to solar UV radiation has been shown to have multiple effects on the immune system $(17,18)$. Suppression of immune reactions after UVB exposure of the skin suggests that immune factors contribute to the pathogenesis of solar UV light-induced skin cancer in mice and probably in humans as well $(2,71)$. Epidemiological data indicate that chronically immunosuppressed patients living in regions of intense sun exposure experience an exceptionally high rate of skin cancer (72). This observation supports the evidence that immune surveillance is an important mechanism designed to prevent the generation and maintenance of neoplastic cells (73). The incidence of skin cancers, particularly squamous cell carcinomas, is also increased among organ transplant recipients (74-77). These studies provide evidence in support of the concept that immune suppression promotes the risk of skin cancer development.

It has been shown that UV-induced infiltrating cells, particularly $\mathrm{MHC}^{+} \mathrm{CD} 11 \mathrm{~b}^{+}$cells (activated macrophages and neutrophils), have a role in UV-induced suppression of contact hypersensitivity (CHS) response (78). CHS is considered as a T-cell mediated immune response. It was observed that prevention of UV-induced suppression of CHS by silymarin was associated with the reduction in infiltration of $\mathrm{MHC}^{+} \mathrm{CD} 11 \mathrm{~b}^{+}$cell type into UVB-irradiated skin sites. The prevention of infiltration of $\mathrm{CD} 11 \mathrm{~b}^{+}$cells was also supported by the fact that silymarin inhibited the activity of myeloperoxidase, a marker of leukocyte infiltration, in the UVB irradiated skin (35). The blocking of infiltrating leukocytes using anti-CD11b antibody or treatment with soluble complement receptor type-I blocked UV-induced immune suppression and tolerance induction in $\mathrm{C} 3 \mathrm{H} / \mathrm{HeN}$ mice $(78,79)$. These observations indicate that prevention of UVinduced suppression of CHS by silymarin is mediated through the suppression of infiltration of $\mathrm{MHC}^{+} \mathrm{CD} 11 \mathrm{~b}^{+}$cell population. Such type of immunological modulation in UV irradiated skin of mouse was also found with the topical treatment of other phytochemicals, such as green tea polyphenols (80). Therefore, it is evident that selected phytochemicals possess the capability of modulating UVinduced immunological responses in in vivo system.

The work in Katiyar's laboratory has shown that topical application of silymarin inhibits UVB radiation-induced inflammatory responses, oxidative stress, and the induction of photocarcinogenesis in mice $(7,35,61)$. It also has been shown that silibinin also inhibits UVB-induced skin photodamage including the inhibition of photocarcinogenesis in mice whether it is applied topically before or after UVB irradiation or given in the diet (59). As UVB-induced immunosuppression has been implicated in the development of 
photocarcinogenesis, further studies with silymarin were conducted in animals using local and systemic models of CHS. Topical application of silymarin inhibits UVB-induced suppression of CHS response to 2,4-dinitrofluorobenzene (DNFB), a skin contact sensitizer, in both local and systemic models of contact hypersensitivity (81) (Fig. 3). Similar results were observed when the effects of silibinin on UVB-induced suppression of the CHS response were examined using identical experimental conditions and models. These data provide first line evidence that prevention of photocarcinogenesis by silymarin may be, at least in part, due to the prevention of UVB-induced immunosuppression in mice.

To define the mechanism by which silymarin inhibits UVB-induced immunosuppression, it was observed that treatment of mice with silymarin inhibits UVB-enhancement of interleukin (IL)-10 levels both in the skin and in the draining lymph nodes $(35,81)$. IL-10 is considered as an immunosuppressive cytokine. It has been shown that intraperitoneal administration of IL-10 inhibits the sensitization of mice to trinitrophenyl-coupled spleen cells in an assay of delayed type hypersensitivity (82), and that intraperitoneal injection of IL-10 resulted in a significant suppression of the ear swelling response in a model of CHS, suggesting that IL-10 has the ability to block the effector phase of $\mathrm{CHS}$ in vivo. It also has been shown that administration of neutralizing antibodies to IL-10 largely inhibited the ability of UV radiation to suppress sensitization to alloantigens (67). In agreement with these observations, it appears that prevention of UVBinduced immunosuppression by silymarin is mediated, at least in part, through the inhibition of UVB-induced increase in IL-10 production in the skin and draining lymph nodes of mice.

Topical treatment of mice with silymarin increased the production of IL-12 in the skin and draining lymph nodes of UVB-irradiated $\mathrm{C} 3 \mathrm{H} / \mathrm{HeN}$ mice. It has been shown that IL-12 has the ability to stimulate the immune system. The role of enhanced levels of IL-12 production in the silymarininhibition of the UVB-induced immunosuppression was confirmed using a local CHS model, which showed that i.p. injection of anti-IL-12 antibody before sensitization resulted in the silymarin-treated mice exhibiting UVB-induced suppression of the CHS response to DNFB. This observation was further supported when CHS experiments were conducted in IL-12 knockout (IL-12 KO) mice and their wild-type counterparts $(\mathrm{C} 3 \mathrm{H} / \mathrm{HeN})$ in which topical application of silymarin failed to prevent UVB-induced immunosuppression in the IL-12 KO mice, but prevented it in wild-type mice. Further, the i.p. injection of recombinant IL-12 to UVB exposed wild-types and IL-12 KO mice both restored CHS response in the mice which support the evidence that IL-12 plays a crucial role in the prevention of UVB-induced immunosuppression in mice.

The immunostimulatory effects of IL-12 have been demonstrated in an in vivo system $(83,84)$ and IL- 12 has been shown to play a role in vivo as a mediator and adjuvant for the induction phase of the CHS response (83). CHS appears to be a Th1 type cell-mediated immune response (85) and the Langerhans cells, which act as critical epidermal antigen presenting cells in the induction phase of CHS (86), have been reported to produce IL-12. After UV exposure, the antigen presenting cells present in the skin migrate to the regional lymph nodes and initiate sensitization. It is possible that silymarin treatment enhances the levels of IL-12 in the draining lymph nodes by increasing the number of antigen presenting cells that migrate from the skin to the regional lymph nodes in UVB-irradiated mice. IL-12 stimulates the development and function of T-cells particularly the development of Th1 type cells by stimulating the production of IFN- $\gamma$ (87-89). Intraperitoneal injection of IL-12 prevents UV-induced suppression of CHS (90) and overcomes UVinduced hapten-specific tolerance in mice (91).

In the CHS model, the silymarin-induced increases in the levels of IL-12 in the draining lymph nodes of the UVBirradiated mice could tilt the immune response in favor of the development of Th1 type cells. Together with the effect of silymarin on the production of IL-10, the silymarin-induced shift in the cytokine balance of IL-10 and IL-12 appears to be a potential mechanism by which silymarin may reverse or inhibit UVB-induced suppression of contact hypersensitivity response in mice. Similar immunomodulatory effects were observed with other phytochemicals, such as topical treatment of (-)-epigallocatechin-3-gallate (80) and polyphenolic components of green tea in drinking water of mice (92), and they seem to share similar effector mechanisms in vivo animal models.

\section{Future prospects of silymarin in skin photoprotection}

The studies conducted in vivo in animal models with silymarin/ silibinin suggest that this phytochemical possesses potent anti-photocarcinogenic ability which is mediated through its anti-inflammatory, anti-oxidant and immunomodulatory activities in UVB-irradiated skin. The use of silymarin in combination with sunscreens or skin care lotions may provide an effective strategy for mitigating the adverse biological effects of solar UV radiation that will lead to the protection of the skin from various skin diseases caused by excessive sun exposure. Further studies are required in human system to determine cellular uptake, distribution and long-term effect of silymarin in the skin for their optimal photoprotection.

\section{Acknowledgements}

The original work reported from Dr Katiyar's laboratory was supported from the funds from USPHS Grant, CA105368 (S.K.K.) and Veterans Administration Merit Review Award (S.K.K.),

\section{References}

1. Miller DL and Weinstock MA: Nonmelanoma skin cancer in the United States: incidence. J Am Acad Dermatol 30: 774-778, 1994.

2. Urbach F: Incidences of nonmelanoma skin cancer. Dermatol Clin 9: 751-755, 1991 .

3. Johnson TM, Dolan OM, Hamilton TA, Lu MC, Swanson NA and Lowe L: Clinical and histologic trends of melanoma. J Am Acad Dermatol 38: 681-686, 1998.

4. Mukhtar $\mathrm{H}$ and Elmets CA: Photocarcinogenesis: Mechanisms, models and human health implications. Photochem Photobiol 63: 355-447, 1996.

5. American Cancer Society: Cancer Facts and Figures, 2001. Publication no. 5008.01. American Cancer Society, Atlanta, GA, 2001 
6. O'Shaughnessy JA, Kelloff GJ, Gordon GB, et al: Treatment and prevention of intraepithelial neoplasia: An important target for accelerated new agent development. Recommendations of the American Association for Cancer Research task force on the treatment and prevention of intraepithelial neoplasia. Clin Cancer Res 8: 314-346, 2002.

7. Katiyar SK, Korman NJ, Mukhtar H and Agarwal R: Protective effects of silymarin against photocarcinogenesis in a mouse skin model. J Natl Cancer Inst 89: 556-566, 1997.

8. Huang R-P, Wu J-X, Fan Y and Adamson ED: UV activates growth factor receptor via reactive oxygen intermediates. J Cell Biol 133: 211-220, 1996.

9. Peus D, Vasa RA, Meves A, Pott M, Beyerle A, Squillac K and Pittelkow MR: $\mathrm{H}_{2} \mathrm{O}_{2}$ is an important mediator of UVB-induced EGF-receptor phosphorylation in cultured keratinocytes. J Invest Dermatol 110: 966-971, 1998.

10. Peus D, Vasa RA, Beyerle A, Meves A, Krautmacher C and Pittelkow MR: UVB activates ERK1/ERK2 and p38 signaling pathways via reactive oxygen species in cultured keratinocytes. J Invest Dermatol 112: 751-756, 1999.

11. Katiyar SK, Afaq F, Azizuddin K and Mukhtar H: Inhibition of UVB-induced oxidative stress-mediated phosphorylation of mitogen-activated protein kinase signaling pathways in cultured human epidermal keratinocytes by green tea polyphenol (-)-epigallocatechin-3-gallate. Toxicol Appl Pharmacol 176: $110-117,2001$

12. Mu D, Hsu DS and Sancar A: Reaction mechanism of human DNA repair excision nuclease. J Biol Chem 271: 8285-8294, 1996.

13. Li G and Ho VC: p53-dependent DNA repair and apoptosis respond differently to high and low dose of ultraviolet radiation. Br J Dermatol 139: 3-10, 1998.

14. Applegate LA, Ley RD, Alcalay J and Kripke ML: Identification of molecular targets for the suppression of contact hypersensitivity by ultraviolet radiation. J Exp Med 170: 1117-1131, 1989.

15. Kripke ML, Cox PA, Alas LG and Yarosh DB: Pyrimidine dimers in DNA initiate systemic immunosuppression in UVirradiated mice. Proc Natl Acad Sci USA 89: 7516-7520, 1992.

16. Hart RW, Setlow RB and Woodhead AD: Evidence that pyrimidine dimers in DNA can give rise to tumors. Proc Natl Acad Sci USA 75: 5574-5578, 1976.

17. Kripke ML: Antigenicity of murine skin tumors induced by UV light. J Natl Cancer Inst 53: 1333-1336, 1974.

18. Kripke ML: Immunological unresponsiveness induced by ultraviolet radiation. Immunol Rev 80: 87-102, 1984.

19. Tyrrell RM: Oxidant, antioxidant status and photocarcinogenesis: the role of gene activation. Photochem Photobiol 63: 380-383, 1996.

20. Godar DE, Wengraitis SP, Shreffler J and Sliney DH: UV doses of Americans. Photochem Photobiol 73: 621-629, 2001.

21. Godar DE: UV doses of American children and adolescents. Photochem Photobiol 74: 787-793, 2001

22. Green A, Williams G, Neale R, et al: Daily sunscreen application and betacarotene supplementation in prevention of basal-cell and squamous-cell carcinomas of the skin: a randomised controlled trial. Lancet 354: 723-729, 1999.

23. Azurdia RM, Pagliaro JA, Diffey BL and Rhodes LE: Sunscreen application by photosensitive patients is inadequate for protection. Br J Dermatol 140: 255-258, 1999.

24. Thompson SC, Jolley D and Marks R: Reduction of solar keratoses by regular sunscreen use. N Engl J Med 329: 1147-1151, 1993.

25. Naylor MF, Boyd A, Smith DW, Cameron GS, Hubbard D and Neldner KH: High sun protection factorsunscreens in the suppression of actinic neoplasia. Arch Dermatol 131: 170-175, 1995.

26. Weinstock MA: Do sunscreens increase or decrease melanoma risk: an epidemiologic evaluation. J Invest Dermatol Symp Proc 4: $97-100,1999$

27. Haywood R, Wardman P, Sanders R and Linge C: Sunscreens inadequately protect against ultraviolet-A-induced free radicals in skin: Implications for skin aging and melanoma? J Invest Dermatol 121: 862-868, 2003.

28. Xu CX, Green A, Parisi A and Parsons PG: Photosensitization of the sunscreen octyl p-dimethylaminobenzoate by UVA in human melanocytes but not in keratinocytes. Photochem Photobiol 73: 600-604, 2001

29. Cross SE, Jiang RY, Benson HAE and Roberts MS: Can increasing the viscosity of formulations be used to reduce the human skin penetration of the sunscreen oxybenzone? J Invest Dermatol 117: 147-150, 2001.
30. Keller KL and Fenske NA: Uses of vitamins A, C, and E and related compounds in dermatology: a review. J Am Acad Dermatol 39: 611-625, 1998.

31. Katiyar SK, Elmets CA, Agarwal R and Mukhtar H: Protection against ultraviolet-B radiation-induced local and systemic suppression of contact hypersensitivity and edema responses in $\mathrm{C} 3 \mathrm{H} / \mathrm{HeN}$ mice by green tea polyphenols. Photochem Photobiol 62: 855-861, 1995.

32. Katiyar SK and Mukhtar H: Green tea polyphenol (-)epigallocatechin-3-gallate treatment to mouse skin prevents UVB-induced infiltration of leukocytes, depletion of antigen presenting cells and oxidative stress. J Leukoc Biol 69: 719-726, 2001.

33. Reeve VE, Bosnic M, Rozinova E and Boehm-Wilcox C: A garlic extract protects from ultraviolet B $(280-320 \mathrm{~nm})$ radiationinduced suppression of contact hypersensitivity. Photochem Photobiol 58: 813-817, 1993.

34. Katiyar SK, Agarwal R and Mukhtar H: Inhibition of tumor promotion in SENCAR mouse skin by ethanol extract of Zingiber Officinale rhizome. Cancer Res 56: 1023-1030, 1996.

35. Katiyar SK: Treatment of silymarin, a plant flavonoid, prevents ultraviolet light-induced immune suppression and oxidative stress in mouse skin. Int J Oncol 21: 1213-1222, 2002.

36. Wang Z, Boudjelal M, Kang S, Voorhees JJ and Fisher GJ: Ultraviolet irradiation of human skin causes functional vitamin A deficiency, preventable by all-trans retinoic acid pre-treatment. Nat Med 5: 418-422, 1999.

37. Mittal A, Elmets CA and Katiyar SK: Dietary feeding of proanthocyanidins from grape seeds prevents photocarcinogenesis in SKH-1 hairless mice: Relationship to decreased fat and lipid peroxidation. Carcinogenesis 24: 1379-1388, 2003.

38. Lee EH, Faulhaber D, Hanson KM, Ding W, Peters S, Kodali S and Granstein RD: Dietary lutein reduces ultraviolet radiationinduced inflammation and immunosuppression. J Invest Dermatol 122: 510-517, 2004.

39. Nowell PC: Mechanism of tumor promotion. Cancer Res 46: 2203-2208, 1986.

40. Katiyar SK and Elmets CA: Green tea polyphenolic antioxidants and skin photoprotection. Int J Oncol 18: 1307-1313, 2001.

41. Young AR: Cumulative effects of ultraviolet radiation on the skin: cancer and photoaging. Semin Dermatol 9: 25-31, 1990.

42. Ames BN: Dietary carcinogens and anticarcinogens. Oxygen radicals and degenerative diseases. Science 221: 1256-1264, 1983.

43. Block G: Micronutrients and cancer: time for action? J Natl Cancer Inst 85: 846-848, 1993.

44. Katiyar SK and Mukhtar H: Tea antioxidants in cancer chemoprevention. J Cell Biochem 27: S59-S67, 1997.

45. Wattenberg LW: Antimutagenesis and Anticarcinogenesis, Mechanisms II. Uroda Y, Shankel DM and Waters MD (eds). Plenum Publishing Corp., New York, NY, pp155-166, 1990.

46. Mereish KA, Bunner DL, Ragland DR and Creasia DA: Protection against microcystin-LR-induced hepatotoxicity by silymarin: biochemistry, histopathology, and lethality. Pharm Res 8: 273-277, 1991.

47. Wagner VH, Diesel P and Seitz M: Chemistry and analysis of silymarin from Silybum marianum (L.) Gaertn. Arzneimittelforschung 24: 466-471, 1974.

48. Wagner H, Horhammer L and Munster R: The chemistry of silymarin (silybin), the active principle of the fruits of Silybum marianum (L.) Gaertn. (Carduus marianus) (L.). Arzneimittelforschung 18: 688-696, 1968.

49. Ferenci P, Dragosics B, Dittrich H, Frank H, Benda L, Lochs H, Meryn S, Base W and Schneider B: Randomized controlled trial of silymarin treatment in patients with cirrhosis of the liver. J Hepatol 9: 105-113, 1989.

50. Singh RP, Tyagi AK, Zhao J and Agarwal R: Silymarin inhibits growth and causes regression of established skin tumors in SENCAR mice via modulation of mitogen-activated protein kinases and induction of apoptosis. Carcinogenesis 23: 499-510, 2002.

51. Bhatia N, Zhao J, Wolf DM and Agarwal R: Inhibition of human carcinoma cell growth and DNA synthesis by silibinin, an active constituent of milk thistle: comparison with silymarin. Cancer Lett 147: 77-84, 1999.

52. Salmi HA and Sarna S: Effect of silymarin on chemical, functional, and morphological alterations of the liver: a doubleblind controlled study. Scand J Gastroenterol 17: 517-521, 1982.

53. Saller R, Meier R and Brignoli R: The use of silymarin in the treatment of liver diseases. Drugs 61: 2035-2063, 2001. 
54. Pepping J: Milk thistle: Silybum marianum. Am J Health Syst Pharm 56: 1195-1197, 1999.

55. Agarwal R, Katiyar SK, Lundgren DW and Mukhtar H: Inhibitory effect of silymarin, an anti-hepatotoxic flavonoid, on 12-O-tetradecanoylphorbol-13-acetate-induced epidermal ornithine decarboxylase activity and mRNA in SENCAR mice. Carcinogenesis 15: 1099-1103, 1994.

56. Chatterjee ML, Katiyar SK, Mohan RR and Agarwal R: Flavonoid antioxidant, silymarin, affords exceptionally high protection against tumor promotion in SENCAR mouse skin tumorigenesis model. Cancer Res 59: 622-632, 1999.

57. Strom S: Basal and squamous cell skin cancers of the head and neck. Weber R, Miller M and Goepfert H (eds). Williams and Wilkins, Baltimore, MD, pp1-7, 1996.

58. Brash DE, Rudolph JA, Simon JA, Lin A, McKenna GJ, Baden HP, Halperin AJ and Ponten J: A role for sunlight in skin cancer: UV-induced p53 mutations in squamous cell carcinoma. Proc Natl Acad Sci USA 88: 10124-10128, 1991.

59. Gu M, Dhanalakshmi S, Singh RP, Agarwal C and Agarwal R: Silibinin protects against photocarcinogenesis via modulation of cell cycle regulators, mitogen-activated protein kinases, and Akt signaling. Cancer Res 64: 6349-6356, 2004.

60. Vayalil PK, Elmets CA and Katiyar SK: Treatment of green tea polyphenols in hydrophilic cream prevents UVB-induced oxidation of lipids and proteins, depletion of antioxidant enzymes and phosphorylation of MAPK proteins in SKH-1 hairless mouse skin. Carcinogenesis 24: 927-936, 2003.

61. Katiyar SK, Meleth S. and Sharma SD: Silymarin, a flavonoid from milk thistle (Silybum marianum L.), inhibits UV-induced oxidative stress through targeting infiltrating $\mathrm{CD} 11 \mathrm{~b}^{+}$cells in mouse skin. Photochem Photobiol 84: 266-271, 2008.

62. Svobodova A, Zdarilova A, Maliskova J, Mikulkova H, Walterova D and Vostalova J: Attenuation of UVA-induced damage to human keratinocytes by silymarin. J Dermatol Sci 46: $21-30,2007$

63. Svobodova A, Zdarilova A, Walterova D and Vostalova J: Flavonolignans from Silybum marianum moderate UVA-induced oxidative damage to HaCaT keratinocytes. J Dermatological Sci 48: 213-224, 2007.

64. Bode AM and Dong Z: Mitogen-activated protein kinase activation in UV-induced signal transduction. Sci STKE 2003: RE2, 2003.

65. Gu M, Dhanalakshmi S, Mohan S, Singh RP and Agarwal R Silibinin inhibits ultraviolet B radiation-induced mitogenic and survival signaling, and associated biological responses in SKH-1 mouse skin. Carcinogenesis 26: 1404-1413, 2005.

66. Black AK, Greaves MW, Hensby CN and Plummer NA: Increased prostaglandins E2 and F2alpha in human skin at 6 and $24 \mathrm{~h}$ after ultraviolet B irradiation (290-320 nm). Br J Clin Pharmacol 5: 431-436, 1978.

67. Rivas JM and Ullrich SE: The role of IL-4, IL-10, and TNF-alpha in the immune suppression induced by ultraviolet radiation. J Leukoc Biol 56: 769-775, 1994.

68. Meeran SM, Akhtar S and Katiyar SK: Inhibition of UVB-induced skin tumor development by drinking green tea polyphenols is mediated through DNA repair and subsequent inhibition of inflammation. J Invest Dermatol 129: 1258-1270, 2009.

69. Nowell PC: Mechanism of tumor promotion. Cancer Res 46: 2203-2208, 1986.

70. Katiyar SK, Afaq F, Perez A and Mukhtar H: Green tea polyphenol (-)-epigallocatechin-3-gallate treatment of human skin inhibits ultraviolet radiation-induced oxidative stress. Carcinogenesis 22: 287-294, 2001

71. Yoshikawa T, Rae V, Bruins-Slot W, vand-den-Berg JW, Taylor JR and Streilein JW: Susceptibility to effects of UVB radiation on induction of contact hypersensitivity as a risk factor for skin cancer in humans. J Invest Dermatol 95: 530-536, 1990.

72. Kinlen L, Sheil A, Peta J and Doll R: Collaborative United Kingdom-Australia study of cancer in patients treated with immunosuppressive drugs. Br Med J 2: 1461-1466, 1979.
73. Burnet FM: Immunological Surveillance. Pergamon Press, Oxford, 1970

74. Ondrus D, Pribylincova V, Breza J, Bujdak P, Miklosi M, Reznicek $\mathbf{J}$ and Zvara $\mathrm{V}$ : The incidence of tumors in renal transplant recipients with long-term immunosuppressive therapy. Int Urol Nephrol 31: 417-422, 1999.

75. Cowen EW and Billingsley EM: Awareness of skin cancer by kidney transplant patients. J Am Acad Dermatol 40: 697-701, 1999.

76. Otley CC and Pittelkow MR: Skin cancer in liver transplant recipients. Liver Transpl 6: 253-262, 2000.

77. Fortina AB, Caforio AL, Piaserico S, Alaibac M, Tona F, Feltrin G, Livi U and Peserico A: Skin cancer in heart transplant recipients: frequency and risk factor analysis. J Heart Lung Transplant 19: 249-255, 2000.

78. Hammerberg C, Duraiswamy N and Cooper KD: Reversal of immunosuppression inducible through ultraviolet-exposed skin by in vivo anti-CD11b treatment. J Immunol 157: 5254-5261, 1996.

79. Hammerberg C, Katiyar SK, Carroll MC and Cooper KD: Activated complement component 3 (C3) is required for ultraviolet induction of immunosuppression and antigenic tolerance. J Exp Med 187: 1133-1138, 1998.

80. Katiyar SK, Challa A, McCormick TS, Cooper KD and Mukhtar H: Prevention of UVB-induced immunosuppression in mice by green tea polyphenol (-)-epigallocatechin-3-gallate may be associated with alterations in IL-10 and IL-12 production. Carcinogenesis 20: 2117-2124, 1999.

81. Meeran SM, Katiyar S, Elmets CA and Katiyar SK: Silymarin inhibits UV radiation-induced immunosuppression through augmentation of interleukin-12 in mice. Mol Cancer Ther 5: $1660-1668,2006$

82. Schwarz A, Grabbe S, Riemann $\mathrm{H}$, et al: In vivo effects of interleukin-10 on contact hypersensitivity and delayed-type hypersensitivity reactions. J Invest Dermatol 103: 211-216, 1994.

83. Muller G, Saloga J, Germann T, Schuler G, Knop J and Enk AH: IL-12 as mediator and adjuvant for the induction of contact sensitivity in vivo. J Immunol 155: 4661-4668, 1995.

84. Trinchieri G: Interleukin-12 and its role in the generation of Th1 cells. Immunol Today 14: 335-338, 1993.

85. Hauser C: Cultured epidermal Langerhans cells activate effector $\mathrm{T}$ cells for contact sensitivity. J Invest Dermatol 95: 436-440, 1990.

86. Toews GB, Bergstresser PR, Streilein JW and Sullivan S: Epidermal Langerhans cell density determines whether contact hypersensitivity or unresponsiveness follows skin painting with DNFB. J Immunol 124: 445-453, 1980

87. Hsieh C, Macatonia SE, Tripp CS, Wolf SF, O'Garra A and Murphy KM: Development of Th1 CD4 ${ }^{+} \mathrm{T}$ cells through IL-12 produced by Listeria-induced macrophages. Science 260: 547-549, 1993.

88. Scott P: IL-12: initiation cytokine for cell-mediated immunity. Science 260: 496-497, 1993

89. Manetti R, Parronchi P, Giudizi MG, Piccinni MP, Maggi E, Trinchieri G and Romagnani S: Natural killer cell stimulatory factor (interleukin-12 [IL-12]) induces T helper type 1 (Th1)specific immune responses and inhibits the development of IL-4producing Th cells. J Exp Med 177: 1199-1204, 1993.

90. Schmitt DA, Owen-Schaub L and Ullrich SE: Effect of IL-12 on immune suppression and suppressor cell induction by ultraviolet radiation. J Immunol 154: 5114-5120, 1995.

91. Schwarz A, Grabbe S, Aragane Y, et al: Interleukin-12 prevents ultraviolet B-induced local immunosuppression and overcomes UVB-induced tolerance. J Invest Dermatol 106: 1187-1191, 1996.

92. Katiyar SK, Vaid M, van Steeg H and Meeran SM: Green tea polyphenols prevent UV-induced immunosuppression by rapid repair of DNA damage and enhancement of nucleotide excision repair genes. Cancer Prev Res (Phila Pa) Jan. 26, 2010 (Epub ahead of print). 